\title{
AUSLANDER-REITEN TRIANGLES IN SUBCATEGORIES
}

\author{
PETER JØRGENSEN
}

\begin{abstract}
This paper introduces Auslander-Reiten triangles in subcategories of triangulated categories.

The main theorem of the paper shows that there is a close connection with covers and envelopes, also known as minimal rightand left-approximations. Namely, under suitable assumptions, if $C$ is an object in the subcategory $\mathrm{C}$ of the triangulated category $\mathrm{T}$ and
\end{abstract}

$$
X \longrightarrow Y \longrightarrow C \longrightarrow
$$

is an Auslander-Reiten triangle in $\mathrm{T}$, then there is an AuslanderReiten triangle

$$
A \longrightarrow B \longrightarrow C \longrightarrow
$$

in $\mathrm{C}$ if and only if there is a $\mathrm{C}$-cover of the form $A \longrightarrow X$.

The main theorem is used to give a new proof of the existence of Auslander-Reiten sequences over finite dimensional algebras.

\section{INTRODUCTION}

Auslander-Reiten sequences, also known as almost split exact sequences, are one of the central tools in the representation theory of Artin algebras. The precise definition can be found in many places; the book [4] gives an excellent presentation. For the purposes of this introduction, the best way to treat these sequences is perhaps to give a sample of their properties.

If

$$
0 \longrightarrow A \longrightarrow B \longrightarrow C \longrightarrow 0
$$

is an Auslander-Reiten sequence in the category of finitely generated modules over an Artin algebra, then $A$ and $C$ are indecomposable modules which determine each other. Conversely, if $A$ or $C$ is a given

2000 Mathematics Subject Classification. 16G70, 18E30.

Key words and phrases. Auslander-Reiten sequence, cover, envelope, finite dimensional algebra, minimal approximation, precover, preenvelope, Wakamatsu's Lemma. 
indecomposable module, then there is an Auslander-Reiten sequence (11). Moreover, the homomorphisms in the sequence determine the socalled irreducible homomorphisms out of $A$ and into $C$, and in good cases, the irreducible homomorphisms between indecomposable modules can be used to determine all module homomorphisms. In effect, the Auslander-Reiten sequences sum up a great deal of information about the module category of an Artin algebra.

The theory of Auslander-Reiten sequences was founded in [3], and in fact, makes sense for abstract abelian categories. Later on, Auslander and Smalø in 5] developed a more general theory of Auslander-Reiten sequences in subcategories; these permit the study of non-abelian subcategories of abelian categories.

Another later advance was Happel's theory of Auslander-Reiten triangles, see [7]; these play the same role in triangulated categories as Auslander-Reiten sequences do in abelian categories.

On this background, it seems very natural to try to develop a theory of Auslander-Reiten triangles in subcategories. Such a theory is given in this paper; it permits the study of non-triangulated subcategories of triangulated categories.

Auslander-Reiten triangles in subcategories are introduced in Definition 1.3. The main theorem I will prove about them is Theorem 3.1. which shows that there is a close connection between Auslander-Reiten triangles in a subcategory and the approximation properties of the subcategory. Namely, let $\mathrm{T}$ be a suitable triangulated category, $\mathrm{C}$ a suitable subcategory. If $C$ is in $C$ and there is an Auslander-Reiten triangle

$$
X \longrightarrow Y \longrightarrow C \longrightarrow
$$

in $\mathrm{T}$, then there is an Auslander-Reiten triangle

$$
A \longrightarrow B \longrightarrow C \longrightarrow
$$

in $\mathrm{C}$ if and only if there is a C-cover, that is, a minimal right-Capproximation, of the form $A \longrightarrow X$; see Definition 1.4, This echoes the properties of Auslander-Reiten sequences in subcategories discovered by Kleiner in [8].

The paper is organized as follows: This introduction ends with some blanket items, including the definition of Auslander-Reiten triangles in subcategories. Section 2 gives some lemmas. Section 3 proves the main theorem, stating the connection between Auslander-Reiten triangles in subcategories and the approximation properties of the subcategory. 
Section 4 uses the main theorem to give a new proof of the existence of Auslander-Reiten sequences over finite dimensional algebras.

The following setup takes place in the world of triangulated categories. For background information, see [7] or [13].

Setup 1.1. Throughout, $k$ is a field and $\mathrm{T}$ is a skeletally small $k$-linear triangulated category with split idempotents in which each Hom space is finite dimensional over $k$.

By $C$ is denoted a full subcategory of $\mathrm{T}$ which is closed under extensions and direct summands. That is, if $A \longrightarrow B \longrightarrow C \longrightarrow$ is a distinguished triangle with $A$ and $C$ in $C$ then $B$ is isomorphic to an object in $\mathrm{C}$, and if $A$ is an object in $\mathrm{C}$ for which $A \cong A_{1} \amalg A_{2}$, then $A_{1}$ and $A_{2}$ are isomorphic to objects in C.

Remark 1.2. By [15, p. 52, thm.], the conditions of Setup 1.1 imply that $\mathrm{T}$ is a Krull-Schmidt category. That is, each object in $\mathrm{T}$ is the coproduct of finitely many indecomposable objects which are unique up to isomorphism.

Definition 1.3. Let $\Sigma$ denote the suspension in T. A distinguished triangle

$$
A \longrightarrow B \longrightarrow C \longrightarrow \Sigma A
$$

with $A, B$, and $C$ in $C$ is called an Auslander-Reiten triangle in $\mathrm{C}$ if it satisfies the following.

(i) The morphism $C \longrightarrow \Sigma A$ is non-zero.

(ii) If $A^{\prime}$ is in $\mathrm{C}$ then each morphism $A \longrightarrow A^{\prime}$ which is not a section has a factorization

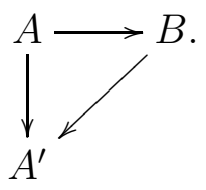

(iii) If $C^{\prime}$ is in $C$ then each morphism $C^{\prime} \longrightarrow C$ which is not a retraction has a factorization

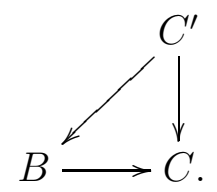

Definition 1.4. Let $X$ be an object of T. A C-precover of $X$ is a morphism $A \longrightarrow X$ with $A$ in $\mathrm{C}$, for which each morphism $A^{\prime} \longrightarrow X$ 
with $A^{\prime}$ in $\mathrm{C}$ has a factorization



A C-cover of $X$ is a C-precover $A \longrightarrow X$ which is right-minimal, that is, satisfies that if $A \longrightarrow A$ is an endomorphism for which the composition $A \longrightarrow A \longrightarrow X$ is equal to $A \longrightarrow X$, then $A \longrightarrow A$ is an isomorphism.

The notions of precover and cover have the dual notions of preenvelope and envelope.

\section{LEMMAS}

This section proves some lemmas which are needed later. The following is a triangulated version of Wakamatsu's Lemma; the proof is not too far from the usual abelian case.

Lemma 2.1 (Triangulated Wakamatsu's Lemma). Suppose that the morphism $A \stackrel{\alpha}{\longrightarrow} X$ in $\mathrm{T}$ is a $\mathrm{C}$-cover and complete it to a distinguished triangle $K \longrightarrow A \stackrel{\alpha}{\longrightarrow} X \longrightarrow$.

Then $\operatorname{Hom}(C, \Sigma K)=0$ for each $C$ in $\mathrm{C}$.

Proof. There is a long exact sequence

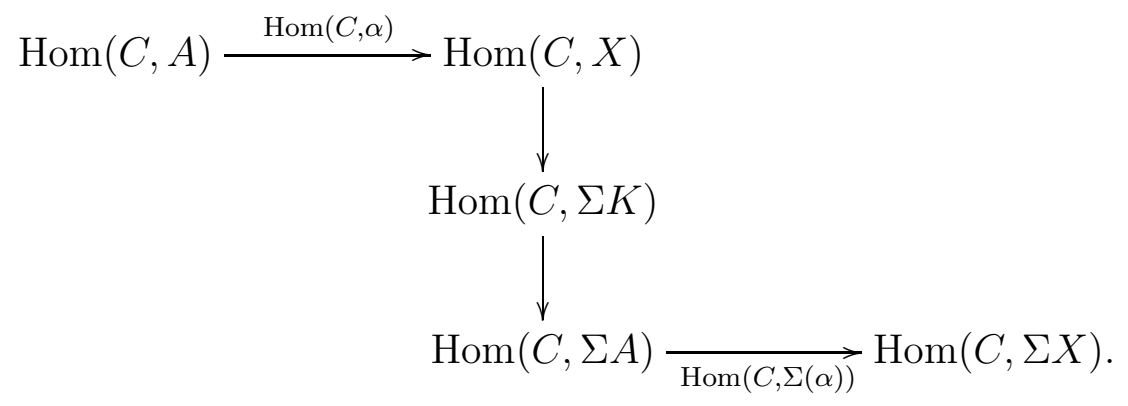

The first map in the sequence is surjective because $A \stackrel{\alpha}{\longrightarrow} X$ is a C-cover, so the second map is zero. I claim that the fourth map in the sequence is injective whence the third map is zero. This forces $\operatorname{Hom}(C, \Sigma K)=0$ as desired. 
To see that the fourth map is injective, let $c$ in $\operatorname{Hom}(C, \Sigma A)$ have $\operatorname{Hom}(C, \Sigma(\alpha))(c)=\Sigma(\alpha) c=0$. This can be interpreted as a commutative square

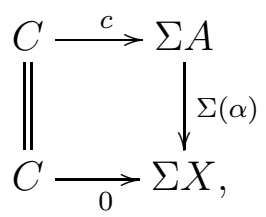

and completing the horizontal morphisms to distinguished triangles gives a commutative diagram

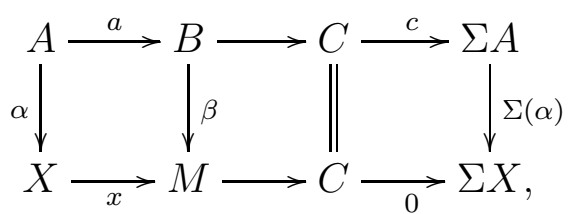

where $\beta$ exists by the axioms of triangulated categories. In particular,

$$
\beta a=x \alpha .
$$

The lower triangle is split so there is a morphism $M \stackrel{m}{\longrightarrow} X$ with

$$
m x=\operatorname{id}_{X} .
$$

Consider the morphism $B \stackrel{m \beta}{\longrightarrow} X$. Since $C$ is closed under extensions, $B$ is in $\mathrm{C}$, and as $A \stackrel{\alpha}{\longrightarrow} X$ is a C-cover, there is a factorization

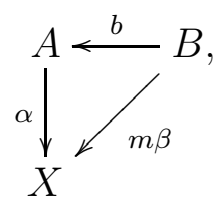

that is,

$$
\alpha b=m \beta .
$$

But now

$$
\alpha \circ b a=m \beta a=m x \alpha=\operatorname{id}_{X} \alpha=\alpha,
$$

and as $A \stackrel{\alpha}{\longrightarrow} X$ is a C-cover, this shows that $A \stackrel{b a}{\longrightarrow} A$ is an automorphism.

Let $a^{\prime}$ be an inverse. Then

$$
\Sigma^{-1}(c)=\operatorname{id}_{A} \Sigma^{-1}(c)=a^{\prime} b a \Sigma^{-1}(c)=a^{\prime} b \circ 0=0,
$$

where I have used that $a \Sigma^{-1}(c)=0$ because $a$ and $\Sigma^{-1}(c)$ are consecutive morphisms in a distinguished triangle. The displayed equation shows $\Sigma^{-1}(c)=0$, and hence $c=0$ as desired. 
Lemma 2.2. Let $X \longrightarrow Y \stackrel{y}{\longrightarrow} Z \stackrel{d}{\longrightarrow} \Sigma X$ be an Auslander-Reiten triangle in $\mathrm{T}$. View the abelian group $\operatorname{Hom}(Z, \Sigma X)$ as a $\operatorname{Hom}(Z, Z)$ right-module via composition of morphisms.

The socle of this module is simple and equal to the submodule generated by $Z \stackrel{d}{\longrightarrow} \Sigma X$.

Proof. Let $M$ be a non-zero submodule of the $\operatorname{Hom}(Z, Z)$-right-module $\operatorname{Hom}(Z, \Sigma X)$. Pick a non-zero element $m$ in $M$, that is, $m$ is a nonzero morphism $Z \longrightarrow \Sigma X$. Then $\Sigma^{-1} Z^{\Sigma^{-1}(m)} \longrightarrow$ is also a non-zero morphism, and it follows from [10, lem. 3.3] that there is a factorization



where the factoring morphism can clearly be taken to be of the form $\Sigma^{-1}(z)$.

Hence $\Sigma^{-1}(d)=\Sigma^{-1}(m) \Sigma^{-1}(z)$ and so $d=m z$. This means that in the $\operatorname{Hom}(Z, Z)$-right-module $\operatorname{Hom}(Z, \Sigma X)$, the element $d$ is a multiple of $m$. Hence $d$ is in $M$ and consequently, the submodule of $\operatorname{Hom}(Z, \Sigma X)$ generated by $d$ is contained in $M$. Since $d$ is non-zero, so is the submodule generated by $d$, and it follows that the socle of $\operatorname{Hom}(Z, \Sigma X)$ is equal to the submodule generated by $d$.

Now note that $\operatorname{Hom}(Z, Z)$ is a local ring by the dual of [9, lem. 2.3]. Since the socle of $\operatorname{Hom}(Z, \Sigma X)$ is generated by a single element, it will follow that it is simple if it is annihilated by the Jacobson radical of $\operatorname{Hom}(Z, Z)$. So let $r$ be in the radical. Then $r$ does not have a rightinverse by the dual of [1, prop. 15.15(e)], and this is the same as to say that the morphism $Z \stackrel{r}{\longrightarrow} Z$ is not a retraction. Hence there is a factorization

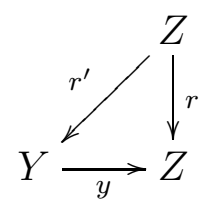

with $r=y r^{\prime}$, and so

$$
d r=d y r^{\prime}=0 \circ r^{\prime}=0
$$

as desired, because $d$ and $y$ are consecutive morphisms in a distinguished triangle whence $d y=0$. 
Lemma 2.3. Let $C$ be in $C$ and let $X \longrightarrow Y \longrightarrow C \stackrel{d}{\longrightarrow} \Sigma X$ be an Auslander-Reiten triangle in $\mathrm{T}$.

Suppose that $A \stackrel{\alpha}{\longrightarrow} X$ is a C-cover. Then $A$ is either zero or indecomposable.

Proof. Suppose that $A$ is not zero. Recall from Remark 1.2 that $\mathrm{T}$ is a Krull-Schmidt category. Let $A_{i}$ be an indecomposable direct summand of $A$, let $A_{i} \longrightarrow A$ be the inclusion of $A_{i}$ into $A$, and denote by $\alpha_{i}$ the composition $A_{i} \longleftrightarrow A \stackrel{\alpha}{\longrightarrow} X$. Since $A \stackrel{\alpha}{\longrightarrow} X$ is a cover, it is clear that $\alpha_{i} \neq 0$. It follows from [10, lem. 3.3] that there is a factorization



Since $d \neq 0$, it follows that $\Sigma^{-1}(d) \neq 0$ and hence $s \neq 0$.

So each indecomposable direct summand $A_{i}$ of $A$ permits a non-zero morphism $\Sigma^{-1} C \longrightarrow A_{i}$. To finish the proof, let me show that at most one indecomposable summand can permit such a morphism:

The C-cover $A \stackrel{\alpha}{\longrightarrow} X$ can be completed to give a distinguished triangle $K \longrightarrow A \stackrel{\alpha}{\longrightarrow} X \longrightarrow$, which can be turned to a distinguished triangle $\Sigma K \longrightarrow \Sigma A \longrightarrow \Sigma X \longrightarrow$. I have $\operatorname{Hom}(C, \Sigma K)=0$ by Lemma 2.1] and hence the homomorphism

$$
\operatorname{Hom}(C, \Sigma A) \longrightarrow \operatorname{Hom}(C, \Sigma X)
$$

is injective. Viewing this as a homomorphism of finite dimensional right-modules over the finite dimensional $k$-algebra $\operatorname{Hom}(C, C)$, the target has simple socle by Lemma 2.2. Hence the image is either zero or indecomposable, and since the homomorphism is injective, the same is true for the source $\operatorname{Hom}(C, \Sigma A)$. So if $A$ splits as $A \cong A_{1} \amalg \cdots \amalg A_{n}$, there can be at most one $i$ for which $\operatorname{Hom}\left(C, \Sigma A_{i}\right) \cong \operatorname{Hom}\left(\Sigma^{-1} C, A_{i}\right)$ is non-zero.

That is, there is at most one indecomposable summand $A_{i}$ of $A$ which permits a non-zero morphism $\Sigma^{-1} C \longrightarrow A_{i}$, as desired.

The easy proof of the following lemma is left to the reader. As a hint, local rings are precisely the rings where the set of elements without a 
left-inverse is closed under addition, and also precisely the rings where the set of elements without a right-inverse is closed under addition, cf. [1, prop. 15.15]. See also [9, lem. 2.3].

Lemma 2.4. Let $A \longrightarrow B \longrightarrow C \longrightarrow$ be an Auslander-Reiten triangle in the subcategory $\mathrm{C}$.

Then $A$ and $C$ have local endomorphism rings. In particular, $A$ and $C$ are indecomposable.

Finally, an elementary fact of linear algebra.

Lemma 2.5. Let $U$ and $V$ be finite dimensional $k$-vector spaces and let

$$
q: U \times V \longrightarrow k
$$

be a bilinear map such that for each non-zero $u$ in $U$, there exists a $v$ in $V$ with $q(u, v) \neq 0$.

Then for each linear map $\varphi: U \longrightarrow k$, there exists a $v$ in $V$ such that

$$
\varphi(-)=q(-, v)
$$

\section{MAIN ThEOREM}

The following is the main theorem of this paper. Recall the triangulated category $T$ and the subcategory $C$ from Setup 1.1.

Theorem 3.1. Let $C$ be in $\mathrm{C}$ and suppose that there exists an $A^{\prime}$ in $\mathrm{C}$ and a non-zero morphism $C \stackrel{c}{\longrightarrow} \Sigma A^{\prime}$.

Let

$$
X \longrightarrow Y \stackrel{y}{\longrightarrow} C \stackrel{d}{\longrightarrow} \Sigma X
$$

be an Auslander-Reiten triangle in $\mathrm{T}$. Then the following are equivalent.

(i) $X$ has a C-cover of the form $A \stackrel{\alpha}{\longrightarrow} X$.

(ii) There is an Auslander-Reiten triangle in $\mathrm{C}$,

$$
A \longrightarrow B \longrightarrow C \longrightarrow \text {. }
$$

Before the proof, let me remark that the existence of a non-zero morphism $C \longrightarrow \Sigma A^{\prime}$ is a necessary condition for the existence of an Auslander-Reiten triangle $A \longrightarrow B \longrightarrow C \longrightarrow$ in $\mathrm{C}$, for if the triangle exists then its connecting morphism $C \longrightarrow \Sigma A$ is non-zero. 
Proof. (i) $\Rightarrow$ (ii). The morphism $C \stackrel{c}{\longrightarrow} \sum A^{\prime}$ is non-zero, so completing it to a distinguished triangle

$$
A^{\prime} \longrightarrow B^{\prime} \stackrel{b^{\prime}}{\longrightarrow} C \stackrel{c}{\longrightarrow} \Sigma A^{\prime}
$$

gives that $b^{\prime}$ is not a retraction. Hence the definition of AuslanderReiten triangles means that $\beta^{\prime}$ exists in the following commutative diagram,

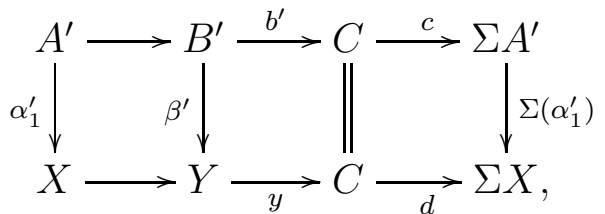

and $\alpha_{1}^{\prime}$ exists by the axioms of triangulated categories. Note that

$$
\Sigma\left(\alpha_{1}^{\prime}\right) c=d .
$$

Since $A \stackrel{\alpha}{\longrightarrow} X$ is a C-cover, there is a factorisation

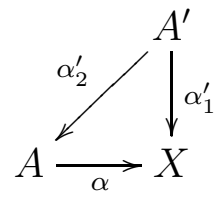

with

$$
\alpha \alpha_{2}^{\prime}=\alpha_{1}^{\prime}
$$

Consider the morphism $C \stackrel{\Sigma\left(\alpha_{2}^{\prime}\right) c}{\longrightarrow} \Sigma A$ and complete it to a distinguished triangle

$$
A \longrightarrow B \stackrel{b}{\longrightarrow} C \stackrel{\Sigma\left(\alpha_{2}^{\prime}\right) c}{\longrightarrow} \Sigma A \text {. }
$$

I claim that this is an Auslander-Reiten triangle in C.

To see so, note first that since $A$ and $C$ are in $\mathrm{C}$, so is $B$, because $\mathrm{C}$ is closed under extensions. Let me next verify that the conditions of Definition 1.3 hold for the distinguished triangle (3).

For condition (i), note that

$$
\Sigma(\alpha) \circ \Sigma\left(\alpha_{2}^{\prime}\right) c=\Sigma\left(\alpha \alpha_{2}^{\prime}\right) c=\Sigma\left(\alpha_{1}^{\prime}\right) c=d,
$$

and since the connecting morphism $d$ of the Auslander-Reiten triangle (2) is non-zero, it follows that $\Sigma\left(\alpha_{2}^{\prime}\right) c \neq 0$.

Observe for later use that in particular, the target $\Sigma A$ of $\Sigma\left(\alpha_{2}^{\prime}\right) c$ must be non-zero, whence also

$$
A \nRightarrow 0
$$


For condition (ii), consider the commutative square

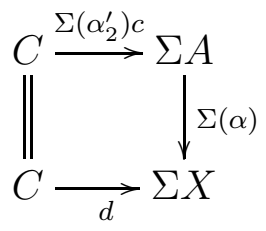

which exists by equation (4). Using the octahedral axiom, this can be extended to a commutative diagram

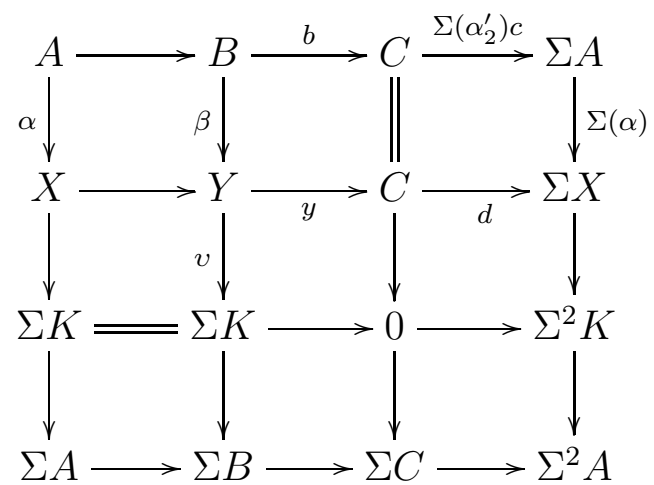

where the first row is the distinguished triangle (31), the second row is the Auslander-Reiten triangle (2), and each row and each column is a distinguished triangle.

Now, given $C^{\prime}$ in $\mathrm{C}$, each morphism $C^{\prime} \longrightarrow C$ which is not a retraction has a factorization

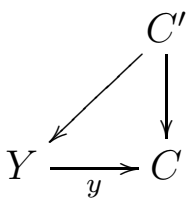

because the second row in the diagram is an Auslander-Reiten triangle. The composition $C^{\prime} \longrightarrow Y \stackrel{v}{\longrightarrow} \Sigma K$ is zero by Lemma 2.1. so the morphism $C^{\prime} \longrightarrow Y$ again has a factorization

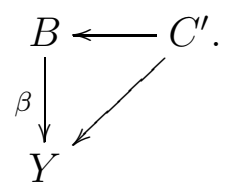

So the morphism $C^{\prime} \longrightarrow C$ has been factored as

$$
C^{\prime} \longrightarrow B \stackrel{\beta}{\longrightarrow} Y \stackrel{y}{\longrightarrow} C,
$$

that is, as $C^{\prime} \longrightarrow B \stackrel{b}{\longrightarrow} C$, verifying condition (ii) of Definition 1.3 
To see that condition (iii) of Definition 1.3 holds for the distinguished triangle (3), note that $A$ is non-zero by equation (5), hence indecomposable by Lemma 2.3, and so $\operatorname{Hom}(A, A)$ is a local ring because $\mathrm{T}$ has finite dimensional Hom spaces and split idempotents.

But then $\operatorname{Hom}(\Sigma A, \Sigma A)$ is also a local ring since it is isomorphic to $\operatorname{Hom}(A, A)$. Consequently, 9, lem. 2.4] implies that in (3), the third morphism $C \stackrel{\Sigma\left(\alpha_{2}^{\prime}\right) c}{\longrightarrow} \Sigma A$ is left minimal, and then [9, lem. 2.5] implies that the second morphism $B \stackrel{b}{\longrightarrow} C$ of (3) is right minimal. Since I have already proved that the distinguished triangle (3) satisfies condition (ii) of Definition [1.3, condition (iii) can now be proved by the same method as in [9, proof of lem. 2.6, $(2) \Rightarrow(3)]$.

(ii) $\Rightarrow$ (i). Let

$$
A \longrightarrow B \stackrel{b}{\longrightarrow} C \stackrel{\mathfrak{d}}{\longrightarrow} \Sigma A
$$

be an Auslander-Reiten triangle in C. Since $\mathfrak{d}$ is non-zero, $B \stackrel{b}{\longrightarrow} C$ is not a retraction, and so by the definition of Auslander-Reiten triangles and the axioms of triangulated categories there is a commutative diagram



in particular,

$$
\alpha \Sigma^{-1}(\mathfrak{d})=\Sigma^{-1}(d) .
$$

I claim that $A \stackrel{\alpha}{\longrightarrow} X$ is a C-cover. In fact, $A$ has local endomorphism ring by Lemma 2.4, so $A \stackrel{\alpha}{\longrightarrow} X$ is right minimal by the dual of [9, lem. 2.4]. Hence it is enough to show that $A \stackrel{\alpha}{\longrightarrow} X$ is a C-precover, cf. Definition 1.4.

To prove this, pick a linear map

$$
\psi: \operatorname{Hom}\left(\Sigma^{-1} C, X\right) \longrightarrow k
$$

which satisfies $\psi\left(\Sigma^{-1}(d)\right) \neq 0$. Given a non-zero $A^{\prime}$ in $\mathrm{C}$, define a bilinear map

$$
\begin{aligned}
q: \operatorname{Hom}\left(\Sigma^{-1} C, A^{\prime}\right) & \times \operatorname{Hom}\left(A^{\prime}, A\right) \longrightarrow k, \\
q\left(s, a^{\prime}\right) & =\psi\left(\alpha a^{\prime} s\right) .
\end{aligned}
$$


Let me first show that Lemma 2.5 applies to $q$. Let $s$ be a non-zero element in $\operatorname{Hom}\left(\Sigma^{-1} C, A^{\prime}\right)$. Construct a distinguished triangle

$$
A^{\prime} \longrightarrow B^{\prime} \stackrel{b^{\prime}}{\longrightarrow} C \stackrel{\Sigma(s)}{\longrightarrow} \Sigma A^{\prime} .
$$

Since C is closed under extensions, $B^{\prime}$ is in C. Since $s$ is non-zero, so is $\Sigma(s)$ and hence $B^{\prime} \stackrel{b^{\prime}}{\longrightarrow} C$ is not a retraction. So there is a factorization



Hence

$$
\mathfrak{d} b^{\prime}=\mathfrak{d} b \beta^{\prime}=0 \circ \beta^{\prime}=0,
$$

where I have used that $\mathfrak{d} b=0$ since $\mathfrak{d}$ and $b$ are consecutive morphisms in a distinguished triangle. So there is a factorization

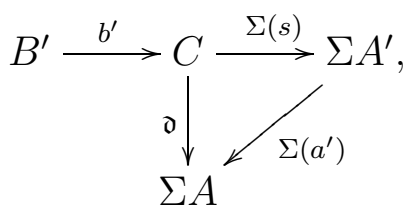

where the factoring morphism can clearly be taken to be of the form $\Sigma\left(a^{\prime}\right)$. Since $\Sigma\left(a^{\prime}\right) \Sigma(s)=\mathfrak{d}$ I get

$$
a^{\prime} s=\Sigma^{-1}(\mathfrak{d})
$$

and so the element $a^{\prime}$ in $\operatorname{Hom}\left(A^{\prime}, A\right)$ satisfies

$$
q\left(s, a^{\prime}\right)=\psi\left(\alpha a^{\prime} s\right)=\psi\left(\alpha \Sigma^{-1}(\mathfrak{d})\right)=\psi\left(\Sigma^{-1}(d)\right) \neq 0,
$$

proving that Lemma 2.5 applies to $q$ as desired.

Now let $A^{\prime} \stackrel{\alpha^{\prime}}{\longrightarrow} X$ be given. I want to factor it through $A \stackrel{\alpha}{\longrightarrow} X$ in order to prove that $A \stackrel{\alpha}{\longrightarrow} X$ is a C-precover. The case $A^{\prime}=0$ is trivial, so suppose $A^{\prime} \neq 0$. Consider the linear map

$$
\begin{gathered}
\varphi: \operatorname{Hom}\left(\Sigma^{-1} C, A^{\prime}\right) \longrightarrow k, \\
\varphi(s)=\psi\left(\alpha^{\prime} s\right) .
\end{gathered}
$$

By Lemma 2.5, there exists an element $a^{\prime}$ in $\operatorname{Hom}\left(A^{\prime}, A\right)$ such that $\varphi(-)=q\left(-, a^{\prime}\right)$, and by the definition of $\varphi$ and $q$, this says

$$
\psi\left(\alpha^{\prime} s\right)=\psi\left(\alpha a^{\prime} s\right) \text { for each } s .
$$


However, 14, proof of prop. I.2.3] shows that the bilinear map

$$
\begin{aligned}
\operatorname{Hom}\left(\Sigma^{-1} C, A^{\prime}\right) \times \operatorname{Hom}\left(A^{\prime}, X\right) \longrightarrow k, \\
(s, \mathfrak{a}) \mapsto \psi(\mathfrak{a} s)
\end{aligned}
$$

is non-degenerate, so equation (6) implies

$$
\alpha^{\prime}=\alpha a^{\prime} \text {. }
$$

That is, I have obtained a factorization

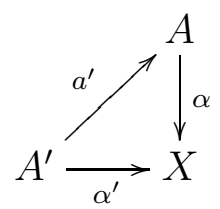

as desired.

The dual result can be proved dually. Let me state it for easy reference.

Theorem 3.2. Let $A$ be in $\mathrm{C}$ and suppose that there exists a $C^{\prime}$ in $\mathrm{C}$ and a non-zero morphism $C^{\prime} \longrightarrow \Sigma A$.

Let

$$
A \longrightarrow Y \longrightarrow Z \longrightarrow
$$

be an Auslander-Reiten triangle in $\mathrm{T}$. Then the following are equivalent.

(i) $Z$ has a C-envelope of the form $Z \longrightarrow C$.

(ii) There is an Auslander-Reiten triangle in $\mathrm{C}$,

$$
A \longrightarrow B \longrightarrow C \longrightarrow \text {. }
$$

\section{Existence of Auslander-Reiten Sequences}

This section uses the results of Section 3 to give a new proof of the existence of Auslander-Reiten sequences over a finite dimensional $k$ algebra $\Lambda$. The idea is to consider the module category $\bmod (\Lambda)$ as a subcategory of a suitable triangulated category of complexes of injective $\Lambda$-modules.

I thank Henning Krause for showing me how to use the methods from [11] and [12 to remove the assumption gldim $\Lambda<\infty$. One of the important tools is the Auslander-Reiten triangles in the homotopy category of complexes of injective $\Lambda$-modules described in 12. Note that 12 already uses these to get Auslander-Reiten sequences; the present section offers an alternative way to do so via the results of Section 3 , 
Lemma 4.1. Let $A \stackrel{\alpha}{\longrightarrow} X$ be a C-precover. Then $A$ has a direct summand $A_{1}$ with inclusion $A_{1} \longleftrightarrow A$ such that the composition

$$
A_{1} \longleftrightarrow A \stackrel{\alpha}{\longrightarrow} X
$$

is a $\mathrm{C}$-cover.

Proof. Consider the category $\mathrm{fp}(\mathrm{T})$ of finitely presented contravariant additive functors from $T$ to the category of abelian groups. This is also known as the Freyd category; see [13, chp. 5] for a survey of the theory.

The category $f p(T)$ is abelian, and the additive functor

$$
\mathrm{T} \longrightarrow \mathrm{fp}(\mathrm{T}), \quad X \longmapsto \operatorname{Hom}(-, X)
$$

permits me to view $T$ as the full subcategory of projective objects of $\mathrm{fp}(\mathrm{T})$.

To get $A_{1}$, view the $\mathrm{C}$-precover $A \stackrel{\alpha}{\longrightarrow} X$ as a morphism in $\mathrm{fp}(\mathrm{T})$ and factor it into an epimorphism followed by a monomorphism,

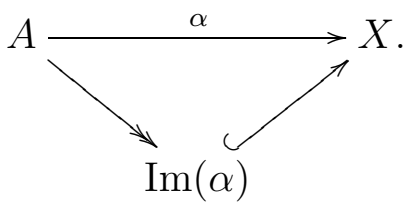

It is not hard to verify that the direct summand $A_{1}$ can be obtained as a projective cover $A_{1} \longrightarrow \operatorname{Im}(\alpha)$ in $\mathrm{fp}(\mathrm{T})$ which exists by [2, thm. 4.12].

Setup 4.2. Let $\Lambda$ be a finite dimensional $k$-algebra over the field $k$.

Consider $\mathrm{K}(\operatorname{Inj} \Lambda)$, the homotopy category of complexes of injective $\Lambda$ left-modules, and $\mathrm{D} \Lambda=\operatorname{Hom}_{k}(\Lambda, k)$, the $k$-linear dual of $\Lambda$ which is a $\Lambda$-bi-module.

Let $\mathrm{T}$ be the full subcategory of $\mathrm{K}(\operatorname{Inj} \Lambda)$ consisting of complexes $X$ for which each $X^{i}$ is finitely generated, and where $\mathrm{H}^{i}(X)=0$ for $i \gg 0$ and $\mathrm{H}^{i}\left(\operatorname{Hom}_{\Lambda}(\mathrm{D} \Lambda, X)\right)=0$ for $i \ll 0$.

Let $C$ be the full subcategory of $T$ which consists of injective resolutions of finitely generated $\Lambda$-left-modules. That is, $\mathrm{C}$ consists of the complexes in $\mathrm{T}$ of the form

$$
\cdots \longrightarrow 0 \longrightarrow A^{0} \longrightarrow A^{1} \longrightarrow \cdots
$$

where all other cohomology groups than $\mathrm{H}^{0}(A)$ are zero. 
Lemma 4.3. The categories $\mathrm{T}$ and $\mathrm{C}$ of Setup 4.2 satisfy the requirements of Setup 1.1.

Proof. It is easy to see that $\mathrm{T}$ is a skeletally small $k$-linear triangulated category.

To see that $\mathrm{T}$ has split idempotents, note first that $\mathrm{K}(\operatorname{Inj} \Lambda)$ does. Namely, by [13, prop. 1.6.8], it is enough to see that $K(\operatorname{Inj} \Lambda)$ has set indexed coproducts, and this is clear because the coproduct of a set indexed family of injective $\Lambda$-left-modules is again injective.

Now let $X$ be in $\mathrm{T}$ and let $X \stackrel{e}{\longrightarrow} X$ be an idempotent. Then there is a splitting of $e$ in $\mathrm{K}(\operatorname{Inj} \Lambda)$, that is, a diagram

$$
X_{1} \stackrel{x_{1}}{\underset{x}{\rightleftarrows}} X
$$

in $\mathrm{K}(\operatorname{Inj} \Lambda)$ with $x_{1} x=e$ and $x x_{1}=\mathrm{id}_{X_{1}}$. By [11, App. B], I can assume that $X_{1}$ is a so-called homotopically minimal complex, and then $x x_{1}=\mathrm{id}_{X_{1}}$ implies that the chain map underlying the homotopy class $x x_{1}$ is invertible. That is, each component of the chain map is bijective, so $X_{1}^{i}$ is isomorphic to a direct summand of $X^{i}$ for each $i$. But each $X^{i}$ is finitely generated by the definition of $\mathrm{T}$, so the same must be true for each $X_{1}^{i}$.

Since it is clear that the direct summand $X_{1}$ also inherits the properties $\mathrm{H}^{i}\left(X_{1}\right)=0$ for $i \gg 0$ and $\mathrm{H}^{i}\left(\operatorname{Hom}_{\Lambda}\left(\mathrm{D} \Lambda, X_{1}\right)\right)=0$ for $i \ll 0$ from $X$, it follows that $X_{1}$ is in T, and so (77) is in fact a splitting of $e$ in T. Hence $\mathrm{T}$ has split idempotents.

To see that $\mathrm{T}$ has finite dimensional Hom spaces over $k$, note that a complex $X$ is the mapping cone of the chain map

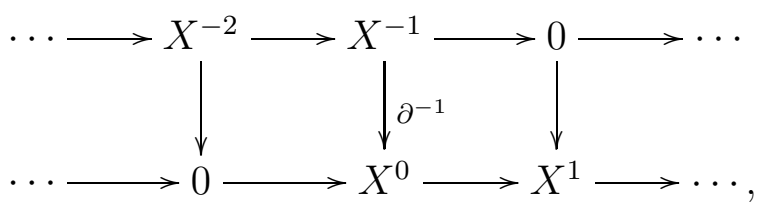

where $\partial$ is the differential of $X$. Using the notation $X^{\sqsupset}$ and $X^{\sqsubset}$ for the two displayed complexes, this implies that there is a distinguished triangle $X^{\sqsupset} \longrightarrow X^{\sqsubset} \longrightarrow X \longrightarrow$ which can be turned to a distinguished triangle

$$
X^{\sqsubset} \longrightarrow X \longrightarrow \Sigma X^{\sqsupset} \longrightarrow \text {. }
$$

If $X$ is in $\top$ then it is easy to check that the complexes $X^{\sqsubset}$ and $\Sigma X^{\sqsupset}$ are also in $T$. So using an obvious notion of left- and right-boundedness for 
complexes, the last triangle shows that each $X$ in $\mathrm{T}$ is the extension of a left-bounded complex $X^{\sqsubset}$ and a right-bounded complex $\Sigma X^{\sqsupset}$, both of which are in $\mathrm{T}$.

Hence it is sufficient to show that Hom spaces between left- and rightbounded complexes in $\mathrm{T}$ are finite dimensional. This is clear for the Hom space from a left-bounded to a right-bounded complex, since the non-zero parts of the complexes only overlap in finitely many degrees, and since the modules in the complexes are finitely generated over $\Lambda$, hence finite dimensional over $k$. The same argument applies to the Hom space from a right-bounded to a left-bounded complex.

If $X$ and $Y$ are both left-bounded complexes in T, then $X$ and $Y$ are left-bounded complexes consisting of injective modules, that is, they are injective resolutions. By the definition of $\mathrm{T}$, the cohomology of $X$ and $Y$ is right-bounded and finitely generated over the finite dimensional $k$-algebra $\Lambda$. This implies that the Hom space $\operatorname{Hom}_{\mathrm{D}(\Lambda)}(X, Y)$ in the derived category $\mathrm{D}(\Lambda)$ is finite dimensional, and so the same is true for $\operatorname{Hom}_{\mathrm{T}}(X, Y)$ since

$$
\operatorname{Hom}_{\mathrm{D}(\Lambda)}(X, Y) \cong \operatorname{Hom}_{\mathrm{K}(\operatorname{Inj} \Lambda)}(X, Y) \cong \operatorname{Hom}_{\mathrm{T}}(X, Y),
$$

where the first $\cong$ is because $Y$ is an injective resolution and the second $\cong$ is because $\mathrm{T}$ is a full subcategory of $\mathrm{K}(\operatorname{Inj} \Lambda)$.

If $X$ and $Y$ are both right-bounded complexes in T, then

$$
P=\operatorname{Hom}_{\Lambda}(\mathrm{D} \Lambda, X) \text { and } Q=\operatorname{Hom}_{\Lambda}(\mathrm{D} \Lambda, Y)
$$

are right-bounded complexes consisting of projective $\Lambda$-left-modules, that is, $P$ and $Q$ are projective resolutions. By the definition of $\mathrm{T}$, the cohomology of $P$ and $Q$ is left-bounded and finitely generated. This implies that $\operatorname{Hom}_{\mathrm{D}(\Lambda)}(P, Q)$ is finite dimensional, and so the same is true for $\operatorname{Hom}_{\mathrm{T}}(X, Y)$ since

$$
\begin{aligned}
\operatorname{Hom}_{\mathrm{D}(\Lambda)}(P, Q) & \stackrel{(\mathrm{a})}{\cong} \operatorname{Hom}_{\mathrm{K}(\Lambda)}(P, Q) \\
& =\operatorname{Hom}_{\mathrm{K}(\Lambda)}\left(\operatorname{Hom}_{\Lambda}(\mathrm{D} \Lambda, X), \operatorname{Hom}_{\Lambda}(\mathrm{D} \Lambda, Y)\right) \\
& \stackrel{(\mathrm{b})}{\cong} \operatorname{Hom}_{\mathrm{K}(\operatorname{Inj} \Lambda)}(X, Y) \\
& \stackrel{(\mathrm{c})}{\cong} \operatorname{Hom}_{\mathrm{T}}(X, Y) .
\end{aligned}
$$

Here $\mathrm{K}(\Lambda)$ denotes the homotopy category of complexes of $\Lambda$-left-modules, (a) is because $P$ is a projective resolution, (b) is because the functor $\left.\operatorname{Hom}_{\Lambda}(\mathrm{D} \Lambda,-)\right)$ is an equivalence from the injective $\Lambda$-left-modules to the projective $\Lambda$-left-modules, and (c) is because $\mathrm{T}$ is a full subcategory of $\mathrm{K}(\operatorname{lnj} \Lambda)$. This shows that $\mathrm{T}$ satisfies the requirements of Setup 1.1 . 
Finally, it is easy to see that $\mathrm{C}$ is closed under extensions and direct summands.

Remark 4.4. $\mathrm{C}$ is the full subcategory of $T$ which consists of injective resolutions of finitely generated $\Lambda$-left-modules. Since the morphisms in $C$ are homotopy classes of chain maps, it is classical that $C$ is equivalent to $\bmod (\Lambda)$, the category of finitely generated $\Lambda$-left-modules. An equivalence can be constructed by letting a module correspond to one of its injective resolutions.

Moreover, let

$$
A \longrightarrow B \longrightarrow C \longrightarrow
$$

be an Auslander-Reiten triangle in the subcategory $\mathrm{C}$ of $\mathrm{T}$, in the sense of Definition 1.3. Then the cohomology long exact sequence gives a short exact sequence

$$
0 \longrightarrow \mathrm{H}^{0}(A) \longrightarrow \mathrm{H}^{0}(B) \longrightarrow \mathrm{H}^{0}(C) \longrightarrow 0
$$

and it is easy to see that this is an Auslander-Reiten sequence in the abelian category $\bmod (\Lambda)$.

Lemma 4.5. $\mathrm{C}$ is a covering class in $\mathrm{T}$.

Proof. By Lemma 4.1 it is enough to show that $\mathrm{C}$ is precovering in $\mathrm{T}$. But given $X$ in $\mathrm{T}$, let $\partial$ be the differential of the complex $X$ and let $A$ be an injective resolution of Ker $\partial^{0}$. It is then easy to check that the canonical chain map

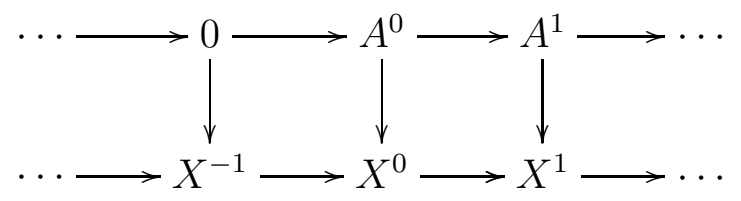

gives a C-precover of $X$ in $\mathrm{T}$.

Theorem 4.6. Let $M$ and $U$ be indecomposable finitely generated $\Lambda$ left-modules and suppose that $M$ is not projective and that $U$ is not injective.

Then there are Auslander-Reiten sequences in $\bmod (\Lambda)$,

$$
0 \longrightarrow K \longrightarrow L \longrightarrow M \longrightarrow 0
$$

and

$$
0 \longrightarrow U \longrightarrow V \longrightarrow W \longrightarrow 0
$$


Proof. It is enough to show that the first of these sequences exists, because the second one can be obtained using the dualization functor $\mathrm{D}(-)=\operatorname{Hom}_{k}(-, k)$.

Since $M$ is not a projective module, there exists a $K^{\prime}$ in $\bmod (\Lambda) \operatorname{such}$ that $\operatorname{Ext}_{\Lambda}^{1}\left(M, K^{\prime}\right) \neq 0$. Let $C$ and $A^{\prime}$ be injective resolutions of $M$ and $K^{\prime}$. Then $C$ and $A^{\prime}$ are in $\mathrm{C}$, and since

$$
\operatorname{Ext}_{\Lambda}^{1}\left(M, K^{\prime}\right) \cong \operatorname{Hom}_{\mathrm{K}(\operatorname{Inj} \Lambda)}\left(C, \Sigma A^{\prime}\right) \cong \operatorname{Hom}_{\mathrm{T}}\left(C, \Sigma A^{\prime}\right),
$$

it follows that there is a non-zero morphism $C \longrightarrow \Sigma A^{\prime}$ in $\mathrm{T}$.

The complex $C$ is a compact object of $\mathrm{K}(\operatorname{Inj} \Lambda)$ by [11, lem. 2.1], and since $M$ is an indecomposable module, $C$ is an indecomposable object of $\mathrm{K}(\operatorname{Inj} \Lambda)$. Hence by [12, thm. 6.3], there is an Auslander-Reiten triangle

$$
\Sigma^{-1} \mathrm{D} \Lambda \otimes_{\Lambda} P \longrightarrow Y \longrightarrow C \longrightarrow
$$

in $\mathrm{K}(\operatorname{Inj} \Lambda)$, where $P$ is a projective resolution of the module $M$.

It is easy to see that $\Sigma^{-1} \mathrm{D} \Lambda \otimes_{\Lambda} P$ is in $\mathrm{T}$, and so (8) is in particular an Auslander-Reiten triangle in $\mathrm{T}$. As $\mathrm{C}$ is covering in $\mathrm{T}$ by Lemma 4.5, there is a C-cover

$$
A \longrightarrow \Sigma^{-1} \mathrm{D} \Lambda \otimes_{\Lambda} P \text {. }
$$

But now Theorem 3.1 says that there is an Auslander-Reiten triangle

$$
A \longrightarrow B \longrightarrow C \longrightarrow
$$

in C, and by Remark 4.4 this gives an Auslander-Reiten sequence

$$
0 \longrightarrow \mathrm{H}^{0}(A) \longrightarrow \mathrm{H}^{0}(B) \longrightarrow \mathrm{H}^{0}(C) \longrightarrow 0
$$

in $\bmod (\Lambda)$. Since $\mathrm{H}^{0}(C) \cong M$, this gives the first Auslander-Reiten sequence claimed in the theorem.

Acknowledgement. I thank Henning Krause for showing me how to use the methods from [11] and [12] to remove the assumption gldim $\Lambda<\infty$ from Section 4, and Karin Erdmann and Vanessa Miemitz for their interest in this work.

\section{REFERENCES}

[1] F. W. Anderson and K. R. Fuller, "Rings and categories of modules", Grad. Texts in Math., Vol. 13, Springer, Berlin, 1974.

[2] M. Auslander, Representation theory of Artin algebras I, Comm. Algebra 1 (1974), 177-268. 
[3] - and I. Reiten, Representation theory of Artin algebras III, Comm. Algebra 3 (1975), 239-294.

[4] _ I. Reiten, and S. O. Smalø, "Representation theory of Artin algebras", Cambridge Stud. Adv. Math., Vol. 36, Cambridge University Press, Cambridge, 1997, first paperback edition with corrections.

[5] _ and S. O. Smalø, Almost split sequences in subcategories, J. Algebra 69 (1981), 426-454.

[6] _ and S. O. Smalø, Addendum to "Almost split sequences in subcategories", J. Algebra 71 (1981), 592-594.

[7] D. Happel, On the derived category of a finite dimensional algebra, Comment. Math. Helv. 62 (1987), 339-389.

[8] M. Kleiner, Approximations and almost split sequences in homologically finite subcategories, J. Algebra 198 (1997), 135-163.

[9] H. Krause, Auslander-Reiten theory via Brown Representability, K-Theory 20 (2000), 331-344.

[10] , Auslander-Reiten triangles and a theorem of Zimmermann, Bull. London Math. Soc. 37 (2005), 361-372.

[11] _ The stable derived category of a noetherian scheme, Compositio Math. 141 (2005), 1128-1162.

[12] _ and J. Le, The Auslander-Reiten formula for complexes of modules, to appear in Adv. Math.

[13] A. Neeman, "Triangulated categories", Ann. of Math. Stud., Vol. 148, Princeton University Press, Princeton, 2001.

[14] I. Reiten and M. Van den Bergh, Noetherian hereditary abelian categories satisfying Serre duality, J. Amer. Math. Soc. 15 (2002), 295-366.

[15] C. M. Ringel, "Tame algebras and quadratic forms", Lecture Notes in Math., Vol. 1099, Springer, Berlin, 1984.

Department of Pure Mathematics, University of Leeds, Leeds LS2 9JT, UNITED KINGDOM

E-mail address: popjoerg@maths.leeds.ac.uk

URL: http://www.maths.leeds.ac.uk/ ${ }^{\sim}$ popjoerg 